\title{
Curcuminoid-loaded poly(methyl methacrylate) nanoparticles for cancer therapy
}

This article was published in the following Dove Press journal:

International Journal of Nanomedicine

\section{Abhispa Sahu' \\ Pratima Solanki² \\ Susmita Mitra'}

'Amity Institute of Nanotechnology, Amity University, Noida, ${ }^{2}$ Special Centre for Nanosciences, Jawaharlal Nehru University, New Delhi, India
Correspondence: Susmita Mitra

Amity Institute of Nanotechnology,

Amity University, Sector-125,

Noida 201304, Uttar Pradesh, India

Tel +9l 9810333880

Emailsmitra@amity.edu

\begin{abstract}
Curcuminoids (Curs), oleoresins from Curcuma longa L., have known anticarcinogenic and anti-inflammatory properties, but high toxicity, poor aqueous solubility and susceptibility to degradation in body fluids are deterrents to their clinical administration. Poly(methyl methacrylate) nanoparticles (PMMA-NPs) are biocompatible and resilient and can entrap hydrophobic drugs. The present investigation is related to solubilizing Curs by incorporating them in these nanoparticles (NPs) and is related to a study comparing the anticarcinogenic effect of drugloaded NPs with free Cur using lung cancer (A549) cell line. Freshly extracted oleoresins were post loaded in PMMA-NPs prepared using emulsion polymerization. The presence of the three components of oleoresins was confirmed by thin-layer chromatography. The size and morphology of void and loaded NPs were determined by dynamic light scattering, scanning electron microscopy and transmission electron microscopy. The NPs were spherical with diameters of $192.66 \pm 5 \mathrm{~nm}$ (void) and 199.16 $\pm 5 \mathrm{~nm}$ (loaded). Drug loading and encapsulation efficiency were $6 \%$ and $93 \%$, respectively. From the Fourier transform infrared spectroscopy spectra, the characteristic absorption vibration of poly(methyl methacrylate) and the bands at $1,383,1,233$ and $962 \mathrm{~cm}^{-1}$ for Cur moiety were observed. Drug release up to 10 days was estimated in buffer, saline and serum. The highest release of $\sim 55 \%$ in $\sim 3$ days was noted in buffer that exhibited the highest bioavailability. The in vitro anticancer activity of loaded drug was evaluated up to 72 hours by MTT assay using A549 cell line. Cellular uptake of dye-loaded NPs was visualized within 30 minutes of incubation. The results revealed that the dose- and time-dependent cell death in case of loaded PMMA-NPs was comparable to that of free Cur. According to the study, the drug-loaded PMMA-NPs appear to be highly suitable for effective, localized and safe chemotherapy.
\end{abstract}

Keywords: polymeric nanoparticle, squamous cell carcinoma, A549 cell line, anticarcinogenic

\section{Introduction}

Cancer therapy using nanomaterials has progressed significantly over the years. Recently, nanoparticles (NPs) have made inroads in the therapy of different types of squamous cell carcinoma (SCC), which is an epithelial malignancy, and the majority of the cases comprise non-melanoma skin cancer, head and neck cancer, oral and esophageal cancer and non-small cell lung cancer. ${ }^{1}$ Surgery, radiation therapy, chemotherapy or combination of these is used to deal with the serious threats of malignancy. Photodynamic therapy is also playing an important role, specifically in superficial $\mathrm{SCC}$, but oral cancer and esophageal cancer need the intervention of localized therapy on exposed lesions. In this context, the use of drug-entrapped NPs can assist in the therapeutic process by controlling the availability of drug at the required site.

Several drugs have been reported for the therapy of carcinoma, of which curcumin, a natural product, has been associated with the regression of pre-malignant lesions 
of the bladder, soft palate, gastrointestinal tract, cervix, lungs and the skin, with treatment responses in established malignancy. ${ }^{2}$ The molecular entity curcuminoid (Cur) is obtained from the rhizome of turmeric. Cur is composed of three fractions (curcumin, demethoxycurcumin and bisdemethoxycurcumin); while all the fractions have reported anticancer activity, demethoxycurcumin has reported additional anti-inflammatory effect ${ }^{3}$ and hence could be used in chemotherapy ensuring dual benefits. However, due to several difficulties in the clinical administration of Cur such as low water solubility and degradation under physiological conditions, its therapeutic efficacy is inadequate. Polymeric nanocarriers with hydrophobic core or shell help in the dissolution of hydrophobic drugs and the preparation of effective safe formulations. ${ }^{4}$ Of the different types of hydrophobic polymers, the biocompatible polyester poly(methyl methacrylate) (PMMA) has been widely used in drug delivery applications since it has some desirable characteristics such as being resistant to chemical hydrolysis, achiral, high permeability for many drugs and lack of toxicity. ${ }^{5}$ In our present investigation, poly(methyl methacrylate) nanoparticles (PMMA-NPs) were considered for loading Cur with dual benefits, primarily due to the fact that these NPs are inert and would be resistant to the $\mathrm{pH}$ and chemical conditions in the vicinity of the superficial and exposed cancerous lesions of the squamous epithelial cells. To further enhance and prolong the drug effect, the surface of the NPs was modified with the bioadhesive polymer poly(acrylic acid) (PAA) to ensure longer residence time of the NPs at the disease site.

\section{Materials and methods}

Methyl methacrylate, PAA, sodium dodecyl sulfate (SDS) and 2,2'-azoisobutyronitrile (AIBN) were purchased from Sigma (St Louis, MO, USA). Chemicals for cell culture and MTT assay were purchased from Sigma. All other chemicals of analytical reagent grade were obtained locally. The cell line A549 was purchased from the cell repository of the National Centre for Cell Science, Pune, India. Cur was extracted by hot solvent method from dried and powdered turmeric, and the presence of all three fractions of the Cur was confirmed by thin-layer chromatography (Figure 1A).
A

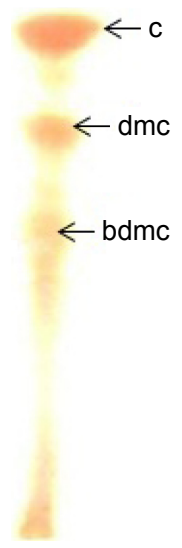

B

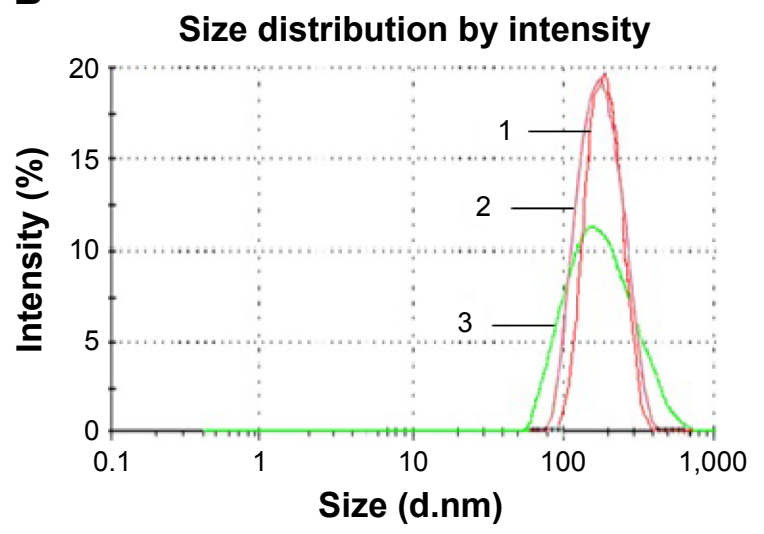

C
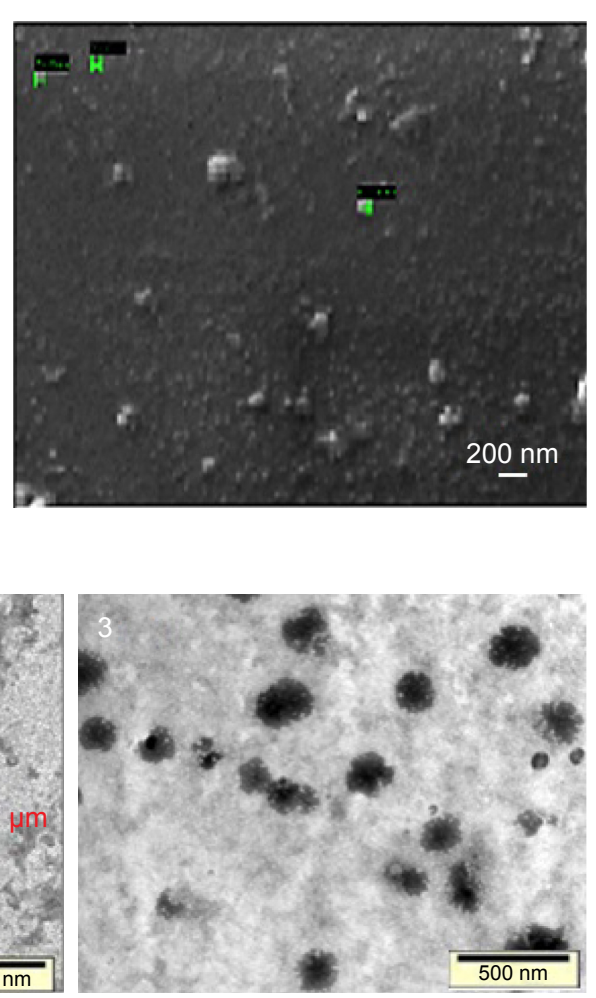

Figure I (A) Thin-layer chromatograph of Cucuma longa extract. (B) DLS of (I) void PMMA-NPs, (2) Cur-loaded PMMA-NPs and (3) PAA-coated Cur-PMMA-NPs. (C) SEM image of loaded PMMA-NPs. (D) TEM image of (I) void PMMA-NPs, (2) loaded PMMA-NPs and (3) PAA-coated Cur-PMMA-NPs.

Abbreviations: DLS, dynamic light scattering; PMMA-NPs, poly(methyl methacrylate) nanoparticles; PAA, poly(acrylic acid); Cur, curcuminoid; SEM, scanning electron microscopy; TEM, transmission electron microscopy; c, curcumin; dmc, demethoxycurcumin; bdmc, bisdemethoxycurcumin. 


\section{NP synthesis by microemulsion polymerization}

A total of $26.6 \mathrm{mg}$ of AIBN and $7.766 \mathrm{mg}$ of SDS were mixed in $20 \mathrm{~mL}$ of Milli-Q water in a three-necked round bottomed flask under constant agitation and nitrogen feed at $70^{\circ} \mathrm{C}$. The monomer was added drop wise, and the above reaction parameters were maintained overnight for the polymerization process.

\section{Drug loading of PMMA-NPs (Cur-PMMA)}

The dispersion of NPs was diluted 10 -fold, and $2 \mathrm{~mL}$ was taken in a dialysis membrane $(12 \mathrm{kD})$ to which Cur was gradually added till free drug was obtained in the membrane bag. The free drug content was estimated from the UV-vis absorption peak at $420 \mathrm{~nm}$ and used to determine loading and encapsulation efficiency using the following equations:

$$
\begin{aligned}
& \text { *Wt of Cur } \\
& \text { in NPs }=\text { Wt of total Cur added }- \text { Wt of free Cur } \\
& \text { Loading efficiency }(\%): \frac{* \text { Wt of Cur in NPs }}{\text { Wt of NPs }} \times 100 \\
& \text { Encapsulation efficiency (\%): } \frac{* \text { Wt of Cur in NPs }}{\text { Wt of total Cur }} \times 100
\end{aligned}
$$

\section{Surface modification with PAA of Cur- loaded PMMA-NPs (PAA-Cur-PMMA)}

Drug-loaded NPs were surface functionalized by adding $0.01 \%(\mathrm{w} / \mathrm{w})$ PAA to NP dispersion under mild stirring and were maintained at $25^{\circ} \mathrm{C}$ for 4 hours.

\section{Characterization techniques used}

Dynamic light scattering (DLS) using Zetasizer Nano ZS90 (Malvern Instruments Ltd, Malvern, UK), scanning electron microscopy (SEM; Zeiss, Oberkochen, Germany), transmission electron microscopy (TEM) and Fourier transform infrared spectroscopy (FTIR; RX1; PerkinElmer Inc., Waltham, MA, USA) were performed for void, loaded and surface-functionalized NPs.

\section{In vitro drug release}

The release was checked in falcon tubes where $1.5 \mathrm{~mL}$ of Cur-PMMA-NP dispersion was added to $1.5 \mathrm{~mL}$ of each of buffer ( $\mathrm{pH}$ 7.4), buffered saline and bovine fetal serum in triplicate and incubated at $37^{\circ} \mathrm{C}$ up to 10 days. After periodic intervals, the samples were centrifuged at $3,000 \mathrm{rpm}$, the supernatant was discarded and the Cur pellet was dissolved in $3 \mathrm{~mL}$ ethanol and the absorbance spectra at $420 \mathrm{~nm}$ were recorded.

\section{Cellular uptake}

To determine the cellular uptake by A549 cell line, cells were placed on a cover slip in a six-well tissue culture plate and incubated at $37^{\circ} \mathrm{C}$ until they reached sub-confluent levels. They were then exposed to $50 \mu \mathrm{g} / \mathrm{mL}$ concentrations of PMMA-NPs, only Nile red dye, Nile red-labeled PMMA-NPs and PAA-PMMA-NPs and determined under fluorescence microscopy.

\section{In vitro cell viability assay}

To determine the effect of free Cur, PMMA-NPs, Cur-PMMANPs and PAA-Cur-PMMA-NPs on cell viability, MTT assay was carried out using lung cancer cell line (A549). For the assay, $2 \times 10^{3}$ cells/well were plated in a 96-well plate and treated with $12.5,25,50$ and $100 \mu \mathrm{g} / \mathrm{mL}$ concentrations of free Cur and equivalent doses of Cur-PMMA-NPs and PAA-Cur-PMMA-NPs; the calculated concentration of PMMA-NPs was also added. The assay was performed for 24, 48 and 72 hours duration, and relative growth inhibition compared to untreated control cells was estimated.

\section{Results and discussion}

NPs were optimized using various reaction parameters, and the resulting void and loaded NPs were sonicated prior to size and morphology determination. The optimum batch of NPs and loaded NPs were of size $192.66 \pm 5 \mathrm{~nm}$ (void) and $199.16 \pm 9 \mathrm{~nm}$ (diameter) as confirmed by DLS and were well dispersed in aqueous media with no aggregates. The size of PAA-Cur-PMMA-NPs was 205 $\pm 7 \mathrm{~nm}$ (Figure 1B). The corresponding loading and encapsulation efficiency of Cur in the nanospheres were found to be $6 \%$ and $93 \%$, respectively. As opposed to free Cur, which exhibits poor aqueous solubility, the loading of the drug in the NPs resulted in high solubility as the encapsulation efficiency of the polymerized particles was very high. According to the SEM image of loaded PMMA-NPs (Figure 1C) and TEM images (Figure 1D) of PMMA-NPs, Cur-PMMA-NPs and PAA-CurPMMA-NPs, the particles were of spherical morphology and of similar size as DLS data. The fuzzy coating was visible following PAA coating.

The physical entrapment of the drug in the polymer was corroborated from the FTIR spectra (data not shown) of the free drug, void polymer matrix and the loaded NPs. The spectrum of Cur-PMMA-NPs revealed the characteristic absorption vibration of PMMA at 1,046 and $840 \mathrm{~cm}^{-1}$, as well as the peak at $1,723 \mathrm{~cm}^{-1}$ that represents the acrylate carboxyl group. Similarly, the bands 2,920,1,461 and $1,024 \mathrm{~cm}^{-1}$, which were due to vibrations of aliphatic $\mathrm{C}-\mathrm{H}$ stretches and 

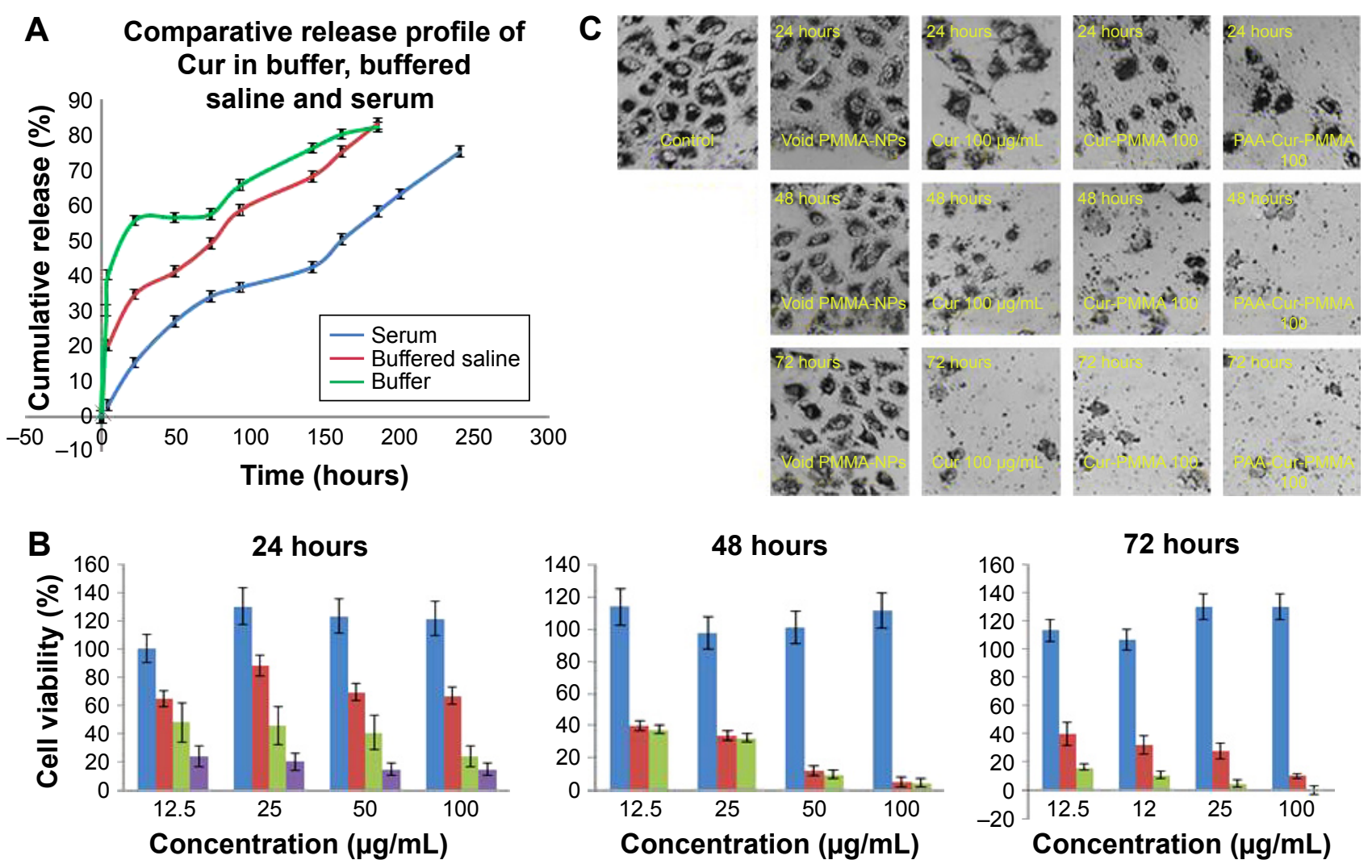

Blank PMMA Free Cur Cur-PMMA PAA-Cur-PMMA

Figure 2 (A) Release profiles of Cur in buffer (green), phosphate buffer saline ( $\mathrm{pH} 7.4$; red) and serum (blue) at $37^{\circ} \mathrm{C}$. (B) Cell viability assay (MTT assay) of void PMMA (blue), Cur (red), Cur-PMMA (green) and PAA-Cur-PMMA (purple) in A549 cell line. All assays were performed in triplicate, and the mean \pm standard deviation is given. $x$-axis represents concentration ( $\mu \mathrm{g} / \mathrm{mL})$, and $y$-axis represents cell viability (\%). (C) Images of A549 cell lines taken using inverted microscope for MTT testing of control, void PMMA, Cur, Cur-PMMA and PAA-Cur-PMMA for 24, 48 and 72 hours duration.

Abbreviations: Cur, curcuminoid; PMMA, poly(methyl methacrylate); PAA, poly(acrylic acid).

mixed vibrations of $\mathrm{CH}_{3}$, aromatic $\mathrm{CCC}$ and $\mathrm{CCH}$ of $\mathrm{Cur}$, were also present in the loaded NPs. The presence of bands at $1,383,1,233$ and $962 \mathrm{~cm}^{-1}$ that represent the in-plane bending of $-\mathrm{OH}$ of the two phenolic and an enolic group, respectively, indicates the presence of the intact Cur moiety in the Cur-PMMA-NPs.

Drug release from PMMA nanospheres in phosphate buffer, buffered saline and serum was analyzed (Figure 2A). Burst release of Cur in buffer (40\%) and buffered saline (20\%) was noted within 5 hours, following which the release was more gradual with $83 \%$ release in 185 hours. In serum, highly sustained and controlled release was observed with $\sim 40 \%$ being released within 150 hours and $75 \%$ in 240 hours.

Cellular uptake results (Figure 3) revealed rapid internalization of Nile red-labeled NP samples as compared to free dye. Within 30 minutes, bright fluorescence was observed within the cells treated with dye-PMMA-NPs and
dye-PAA-PMMA-NPs, while in comparison with free dye, very low fluorescence was visualized. Control cells with blank NPs showed no fluorescence.

The in vitro anticarcinogenic activity of surfacefunctionalized drug-loaded NPs, only drug-loaded NPs, free drug and void NPs was evaluated up to 72 hours. The results revealed that dose- and time-dependent cell death was much higher within a given time duration in case of loaded PMMA-NPs as compared to free Cur. PAA-Cur-PMMA-NPs showed maximum cell death within 24 hours with complete cell death beyond 24 hours (Figures 2B and C).

\section{Conclusion}

In this study, the PMMA-NPs exhibited high drug loading and encapsulation efficiency. The sustained release of Cur in serum can result in a consistent availability of the drug at the required site. The uptake of drug-loaded NPs would ensure intracellular release, thus fast and efficient cell death. 

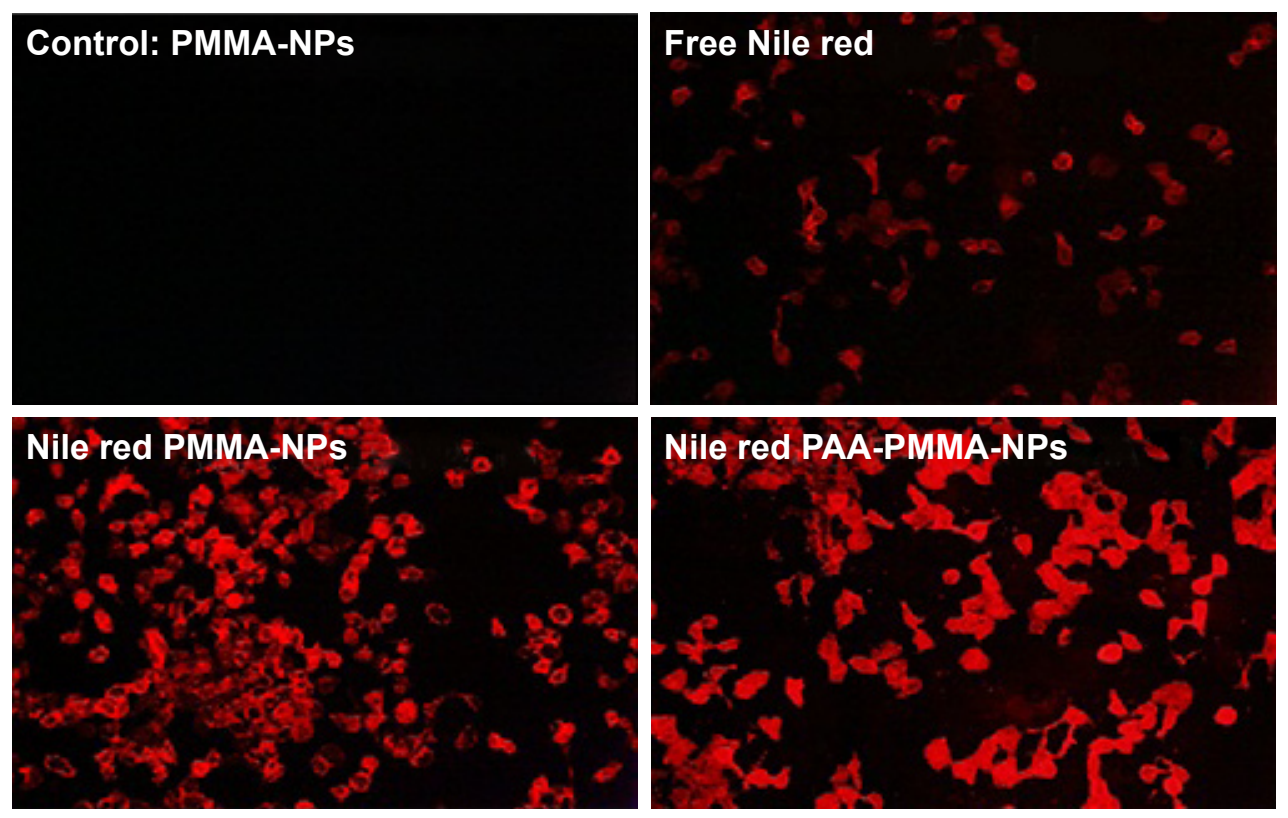

Figure 3 Fluorescence image of the cellular uptake of free Nile red dye and dye-entrapped NPs by A549 cells following 30 minutes of incubation. Abbreviations: NPs, nanoparticles; PMMA, poly(methyl methacrylate); PAA, poly(acrylic acid).

The cell viability assays also demonstrated better affectivity of loaded NPs in comparison to free Cur. According to the study, the NP formulation appears to be highly suitable for localized and safe chemotherapy.

\section{Acknowledgment}

The authors thank Dr RP Singh and Dr LM Bharadwaj for their kind support throughout the project.

\section{Disclosure}

The authors report no conflicts of interest in this work.

\section{References}

1. Wang ZQ, Liu K, Huo ZJ, et al. A cell-targeted chemotherapeutic nanomedicine strategy for oral squamous cell carcinoma therapy. J Nanobiotechnology. 2015;13(63):1-10.
2. Bansal SS, Goel M, Aqil F, Vadhanam MV, Gupta RC. Advanced drug delivery systems of curcumin for cancer chemoprevention. Cancer Prev Res (Phila). 2011;4(8):1158-1171.

3. Guo LY, Cai XF, Lee JJ, et al. Comparison of suppressive effects of demethoxycurcumin and bisdemethoxycurcumin on expressions of inflammatory mediators in vitro and in vivo. Arch Pharm Res. 2008; 31(4):490-496

4. Mukherjee A, Vishwanatha JK. Formulation, characterization and evaluation of curcumin-loaded PLGA nanospheres for cancer therapy. Anticancer Res. 2009;29(10):3867-3876.

5. Elvira C, Fanovich A, Fernandez M, Fraile J, San Román J, Domingo C. Evaluation of drug delivery characteristics of microspheres of PMMAPCL-cholesterol obtained by supercritical-CO2 impregnation and by dissolution-evaporation techniques. J Control Release. 2004;99(2): 231-240.
International Journal of Nanomedicine

\section{Publish your work in this journal}

The International Journal of Nanomedicine is an international, peerreviewed journal focusing on the application of nanotechnology in diagnostics, therapeutics, and drug delivery systems throughout the biomedical field. This journal is indexed on PubMed Central, MedLine, CAS, SciSearch ${ }^{\circledR}$, Current Contents ${ }^{\circledR} /$ Clinical Medicine,

\section{Dovepress}

Journal Citation Reports/Science Edition, EMBase, Scopus and the Elsevier Bibliographic databases. The manuscript management system is completely online and includes a very quick and fair peer-review system, which is all easy to use. Visit http://www.dovepress.com/ testimonials.php to read real quotes from published authors. 